\title{
Protective effects of recombinant human brain natriuretic peptide on the myocardial injury induced by acute carbon monoxide poisoning
}

\author{
Na Li", Xun Gao", Weizhan Wang, Pu Wang, Baoyue Zhu \\ Department of Emergency, Harrison International Peace Hospital Affiliated to Hebei Medical University, Hengshui, China \\ Contributions: (I) Conception and design: N Li, X Gao; (II) Administrative support: N Li, X Gao; (III) Provision of study materials or patients: W \\ Wang, P Wang, B Zhu; (IV) Collection and assembly of data: W Wang, P Wang, B Zhu; (V) Data analysis and interpretation: N Li, X Gao, W \\ Wang, P Wang, B Zhu; (VI) Manuscript writing: All authors; (VII) Final approval of manuscript: All authors. \\ "These authors contributed equally to this work. \\ Correspondence to: Xun Gao, MD. Department of Emergency, Harrison International Peace Hospital Affiliated to Hebei Medical University, 180 East \\ Renmin Road, Hengshui 053000, China. Email: hysysdyl@163.com.
}

Background: To investigate the protective effect of recombinant human brain natriuretic peptide (rhBNP) on myocardial injury after acute carbon monoxide poisoning (ACOP).

Methods: We retrospectively reviewed medical records of patients with ACOP and high risk of cardiac events admitted to our hospital, and grouped them into rhBNP group and control group according treatments they received. Patients in control group received conventional treatment while those in rhBNP group were treated with rhBNP intravenously for 72 hours on the basis of conventional treatment. Levels of amino-terminal pro-brain natriuretic peptide (NT-proBNP), cardiac troponin I (cTnI), serum creatine kinase MB fraction (CK-MB), aldosterone (ALD), angiotensin II (AT II), and endothelin-1 (ET-1) prior to and after treatment of rhBNP or conventional treatment were collected. Corrected QT dispersion (QTcd) results were calculated based on the electrocardiography data. The left ventricular end diastolic diameter (LVEDD), interventricular septal thickness (IVS), left ventricular ejection fraction (LVEF), and stroke output (SV) were measured using color Doppler echocardiography. Major adverse cardiovascular events (MACEs) that occurred within 1 month after treatment were recorded.

Results: A total of 135 patients in the rhBNP group and 136 patients in the control group were enrolled. Baseline characteristics between the two groups were similar at admission. Levels of cTnI, CK-MB, and ET-1 in the rhBNP group were significantly lower than those in the control group at day 1, 2 and 3 after treatment $(\mathrm{P}<0.05)$. Compared with the control group, levels of QTcd, ALD and AT II in the rhBNP group were significantly lower at day 3 after treatment $(\mathrm{P}<0.05)$. After 7 days of treatment, the reduction of NTproBNP in the rhBNP group was significantly greater than that in the control group at each day $(\mathrm{P}<0.05)$, and LVEF, SV and LVEDD in the rhBNP group were all greater than those in the control group. After 1 month of treatment, the incidence of MACEs in the rhBNP group was significantly lower than that in the control group.

Conclusions: For patients with ACOP and high risk of cardiac events, early treatment of rhBNP can protect injured cardiomyocytes, prevent the injury of carbon monoxide on heart, and reduce the incidence of MACE.

Keywords: Acute carbon monoxide poisoning (ACOP); recombinant human brain natriuretic peptide (rhBNP); major adverse cardiac events

Submitted Jun 30, 2020. Accepted for publication Nov 08, 2020.

doi: $10.21037 / \mathrm{cdt}-20-591$

View this article at: http://dx.doi.org/10.21037/cdt-20-591 


\section{Introduction}

Inhalation of excessive carbon monoxide (CO) in a closed environment can cause $\mathrm{CO}$ poisoning. Acute carbon monoxide poisoning (ACOP) presents with a rapid onset, high mortality and disability rate (1). In the early stage of ACOP, the main symptoms are disorders of central nervous system, but the injury of myocardial is often ignored.

The incidence of myocardial injury is positively correlated with the severity of $\mathrm{CO}$ poisoning and the coma time. The mild symptoms of myocardial injury caused by ACOP are abnormal enzymology, low ST level and mild arrhythmia, while the severe symptoms are acute myocardial infarction (AMI), heart failure, malignant arrhythmia, cardiogenic shock and even sudden death (2). The peak of these symptoms occurred in the first three days after poisoning which seriously affected the treatment and prognosis of ACOP.

As we all know, $\mathrm{CO}$ enters the human body to form a large amount of carboxyhemoglobin, which limits the oxygen carrying capacity of the body. Hypoxia can cause metabolic disorder of cardiovascular endothelial cells, stimulate the release of endothelin into the blood, and lead to the injury of myocardial cells (3). Meanwhile, CO can stimulate the sympathetic nerve, produce a large amount of catecholamine, cause blood vessel contraction and heart rate acceleration, increase the burden of heart, and lead to the decline of heart function. A large amount of oxygen free radicals is produced during myocardial hypoxia reperfusion, which make myocardial cells denatured and edematous, and affect cardiac repolarization. Together with the effect of catecholamine, ACOP could lead to arrhythmia and conduction disorder (4). These injuries are often reversible, but during the repairmen of injury, patients may appear a variety of symptoms which may be life-threatening. Therefore, it is an unmet medical need to develop an effective treatment option to prevent ACOP-related myocardial injury.

Brain natriuretic peptide (BNP) was first found in pig brain by Sudoh et al. (5) The secretion of BNP is closely related to the pressure and volume load of the heart. When the heart load increases and the tension of the ventricular wall increases, BNP will erupt into synthesis. It can dilate the peripheral blood vessels, antagonize the renin-angiotensinaldosterone system (RAAS) and sympathetic nervous system (SNS), relief the load of the heart and improve the heart function. BNP can also inhibit the proliferation of vascular smooth muscle and myocardial fibrosis, improve ventricular remodeling and conduction disorders, thus protecting hypoxic cardiomyocytes (6-8). These effects counteract the myocardial injury caused by $\mathrm{CO}$.

Recombinant human BNP (rhBNP), synthesized by artificial recombination technology, has the same biological activity as endogenous BNP and can significantly improve the symptoms and hemodynamic state of heart failure (9-11). However, few studies reported the application of rhBNP in treating patients with ACOP. Therefore, in this study, we evaluated the effects of rhBNP on cardiovascular function indicators and major adverse cardiovascular events (MACEs) of patients with ACOP and high risk of cardiac events. The authors present the study in accordance with the STROBE reporting checklist (available at http://dx.doi.org/10.21037/ cdt-20-591).

\section{Methods}

\section{Patients}

In this study, we retrospectively reviewed the medical records of 271 patients with ACOP and high risk of cardiac events who admitted to our department between November 2017 to February 2020. The diagnosis and poisoning grade classification of ACOP was performed according to Diagnostic Criteria of Occupational Acute Carbon Monoxide Poisoning GBZ23-2002 (12). At least three of the following conditions should be met for the identification of high risk of cardiac events: previous history of coronary heart disease, cardiomyopathy, diabetes mellitus, and hypertension of grade 3; cardiac troponin I (cTnI)-positive; serum creatine kinase $\mathrm{MB}$ fraction $(\mathrm{CK}-\mathrm{MB}) \geq 2$ times of the upper limit of normal level; amino-terminal proBNP (NT-proBNP) >300 pg/mL; abnormal corrected QT dispersion (QTcd); and ST segment depression or T wave depression or inversion. Other inclusion criteria included: within 12 hours after the onset of ACOP (or more than 12 hours after the onset of persistent ischemic symptoms), 18-75 years old, either gender, and patients who provided written informed consent. The exclusion criteria included: malignant arrhythmias such as ventricular tachycardia, ventricular flutter, ventricular fibrillation, or grade 3 atrioventricular block at admission; shock or insufficiency of blood volume; systolic blood pressure $\leq 90 \mathrm{mmHg}$ after the use of vasopressor agents; complications (perforation of ventricular septum, rupture of the mitral chordae tendineae); previous cardiac and renal dysfunction; previous history of coronary artery bypass surgery; suspected aortic 
dissection; cor pulmonale or hypertrophic obstructive cardiomyopathy; could not complete rhBNP treatment for 3 days; or refused to participate in the study.

The study was conducted in accordance with the Declaration of Helsinki (as revised in 2013). The study was approved by institutional ethics committee board of Harrison International Peace Hospital (Number: 2017-0-118) and informed consent was taken from all the patients.

\section{Procedure}

Patients in the control group were given oxygen inhalation and hyperbaric oxygen treatment, and received mannitol and dexamethasone treatment to prevent and treat brain edema, oxiracetam to nourish brain cells, low molecular weight heparin to anticoagulate, and cytochrome $\mathrm{c}$ to improve tissue oxygen supply. If chest distress, chest pain or myocardial ischemia detected by electrostatic grammar occurs, patients should be given aspirin for antiplatelet, atorvastatin. If arrhythmia occurs, metoprolol, digitalis, amiodarone, atropine, and isoprenaline should be given. If there is ventricular fibrillation or ventricular flutter, the cardioversion should be used. In case of heart failure, the patient should be controlled for liquid intake, given diuretics, sodium nitroprusside, and digitalis treatment. Patient with cardiac arrest should be given cardiopulmonary resuscitation.

In the rhBNP group, patients were given rhBNP treatment on the basis of the treatment of control group. RhBNP at a dose of $0.01 \mathrm{~kg} / \mathrm{kg} / \mathrm{min}$ was continuously pumped into the vein for 72 hours. If the blood pressure decreased to below $90 / 60 \mathrm{mmHg}$ in the course of medication, the rehydration or dopamine pressure was given as appropriate. After the medical intervention, the patients whose blood pressure was still unstable should be discontinued rhBNP treatment and were excluded from the study.

\section{Assessment}

At the time of admission, the grade of poisoning and Acute Physiology and Chronic Health Enquiry (APACHE) II score of all patients were recorded; $3 \mathrm{~mL}$ of venous blood was taken to detect NT-proBNP, cTnI and CKMB using bedside dry fluorescent immunoanalyzer (Micropoint Biotechnology Co., Ltd., Shenzhen, China) at the time of admission, day 1, 2 and 3 after treatment. The daily detection of NT-proBNP was extended to day 7 .
Aldosterone (ALD), angiotensin II (AT II), and endothelin-1 (ET-1) were measured using radioimmunoassay kit (Jingkang Bio, Shanghai, China).

For the electrocardiography, the QT interval and RR interval of at least 8 leads were measured at admission and day 3 after treatment. The longest QTc and the shortest QTc interval were calculated according to the correction formula $\mathrm{QTc}=\mathrm{QT} /(\mathrm{RR} \times 0.5)$. QTcd, the difference between the longest QTc and the shortest QTc interval, was calculated and recorded.

After 3 days of treatment, the patients were examined by color Doppler echocardiography using Affiniti 70 (Philips Ultrasound, Bothell, WA, USA). The left ventricular end diastolic diameter (LVEDD), interventricular septal thickness (IVS), left ventricular ejection fraction (LVEF), and stroke output (SV) were recorded. Three cardiac cycles were monitored continuously and the mean values of these parameters were recorded.

MACEs occurred within 1 month after treatment were recorded: AMI; acute left heart failure; malignant arrhythmia including ventricular fibrillation, ventricular flutter, multiform ventricular premature beat, ventricular tachycardia, grade 3 atrioventricular block, and severe sinus arrest; and sudden cardiac death (13).

\section{Statistical analysis}

SPSS 23.0 software was used for statistical analysis. The quantitative data were expressed by mean \pm standard deviation (SD). The data between groups were compared by $t$-test, and the statistical data were compared by $\chi^{2}$ test, A $\mathrm{P}<0.05$ was considered as statistically significant.

\section{Results}

\section{Baseline characteristics}

A total of 271 patients were screened and enrolled in this study, including 135 patients in rhBNP group and 136 patients in control group. Baseline characteristics were presented in Table 1. There was no significant difference in age, gender, severity of poisoning, APACHE II score, NT-proBNP, cTnI, and CK-MB between the rhBNP and control group. ET-1 level in rhBNP group was a little bit higher than that of control group $(\mathrm{P}=0.049)$. The mean age of patients was $46.53 \pm 2.39$ years in rhBNP group and $48.19 \pm 2.68$ years in control group. Six-five-point-onenine percent $(88 / 135)$ of patients in the rhBNP group and 
Table 1 Baseline characteristics

\begin{tabular}{|c|c|c|c|}
\hline Characteristics & rhBNP group $(n=135)$ & Control group $(n=136)$ & $P$ value \\
\hline \multicolumn{4}{|l|}{ Gender, n (\%) } \\
\hline Male & $88(65.19)$ & $81(59.56)$ & 0.32 \\
\hline Female & $47(34.81)$ & $55(40.44)$ & 0.31 \\
\hline Mild & $8(5.93)$ & $10(7.35)$ & 0.62 \\
\hline Moderate & $34(25.19)$ & $42(30.88)$ & 0.31 \\
\hline Severe & $93(68.89)$ & $84(61.76)$ & 0.25 \\
\hline APACHE II score (mean \pm SD) & $14.65 \pm 1.85$ & $15.28 \pm 1.32$ & 0.52 \\
\hline CK-MB (U/L) (mean \pm SD) & $48.32 \pm 11.28$ & $47.56 \pm 13.17$ & 0.39 \\
\hline ET-1 $(\mathrm{pg} / \mathrm{mL})($ mean $\pm \mathrm{SD})$ & $72.56 \pm 15.38$ & $70.15 \pm 16.52$ & 0.049 \\
\hline \multicolumn{4}{|c|}{ Echocardiographic parameters (mean $\pm \mathrm{SD}$ ) } \\
\hline $\operatorname{LVEF}(\%)$ & $57.93 \pm 3.21$ & $57.19 \pm 5.10$ & 0.51 \\
\hline SV (mL) & $73.68 \pm 6.09$ & $74.59 \pm 5.87$ & 0.34 \\
\hline LVEDD (mm) & $48.01 \pm 3.59$ & $47.47 \pm 4.78$ & 0.31 \\
\hline IVS (mm) & $9.50 \pm 0.91$ & $9.40 \pm 0.79$ & 0.29 \\
\hline
\end{tabular}

rhBNP, recombinant human brain natriuretic peptide; SD, standard deviation; APACHE II, Acute Physiology and Chronic Health Enquiry II; NT-proBNP, amino-terminal pro-brain natriuretic peptide; cTnl, cardiac troponin I; CK-MB, creatine kinase MB fraction; ET-1, endothelin-1; LVEF, left ventricular ejection fraction; SV, stroke output; LVEDD, left ventricular end diastolic diameter; IVS, interventricular septal thickness.

$59.56 \%(81 / 136)$ patients in the control group were male. In the rhBNP group, $25.19 \%(34 / 135)$ of patients were moderate poisoning and $68.89 \%(93 / 135)$ of patients were severe poisoning. In the control group, $30.88 \%(42 / 136)$ of patients were moderate poisoning and $61.76 \%(84 / 136)$ of patients were severe poisoning. The APACHE II score in the rhBNP group and control group were $14.65 \pm 1.85$ and $15.28 \pm 1.32$, respectively.

\section{Comparisons of cTnI, CK-MB, and ET-1 level between rbBNP and control group}

No significant difference was observed between rhBNP and control group in terms of cTnI, CK-MB, and ET-1 level at hospital admission. The levels of cTnI, CK-MB, and ET-1 in the rhBNP group were lower than those in the control group at day 1,2 and 3 after treatment, and there was statistical significance between the two groups at each timepoint $(\mathrm{P}<0.05$, Figure 1). After treatment, the cTnI level at day 1,2 and 3 was also significantly lower than that at admission (Figure 1A). CK-MB level increased slightly at day 1 , and then began to decrease at day 2 . At day 2 and 3 timepoints, CK-MB decreased significantly compared with that at admission $(\mathrm{P}<0.05$, Figure $1 B)$. The level of ET-1 in the control group was significantly lower than that at admission at day 2 and 3, and that in the study group was significantly lower than that at admission at day 1,2 and 3 $(\mathrm{P}<0.05$, Figure $1 C)$.

\section{Comparisons of $Q T c d, A L D$, and AT II between rbBNP and control group}

There was no significant difference in the levels of QTcd, ALD and AT II between the rhBNP and control group 
A
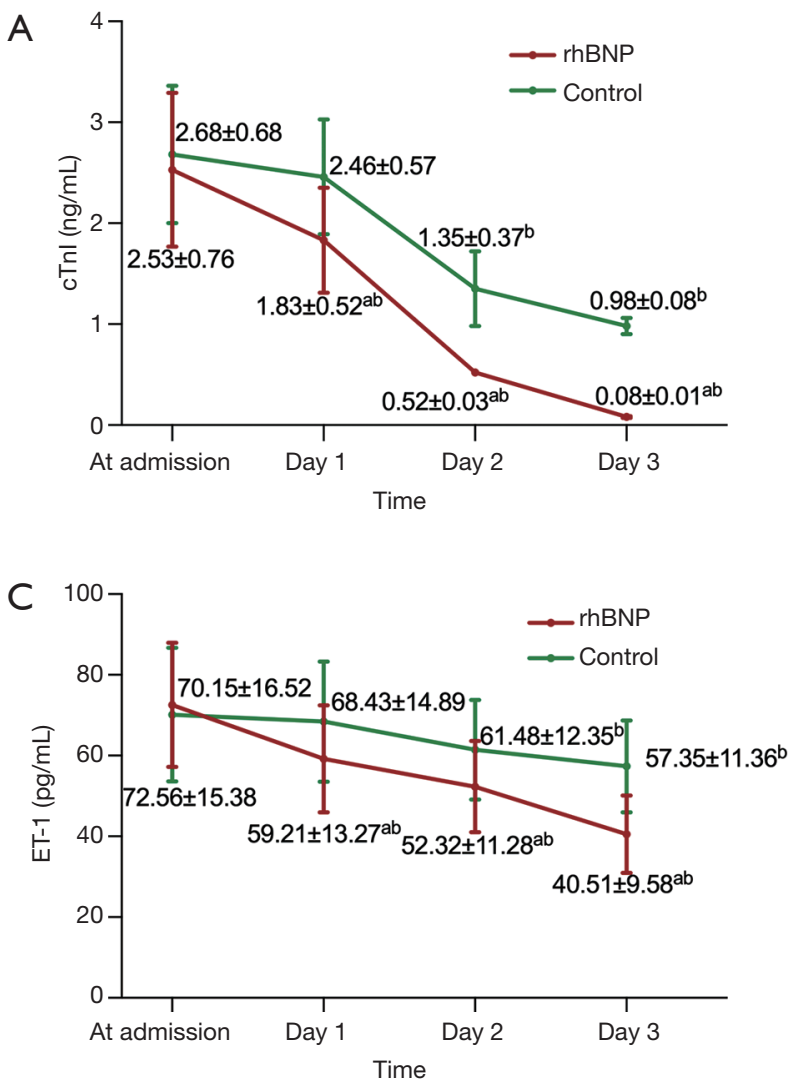

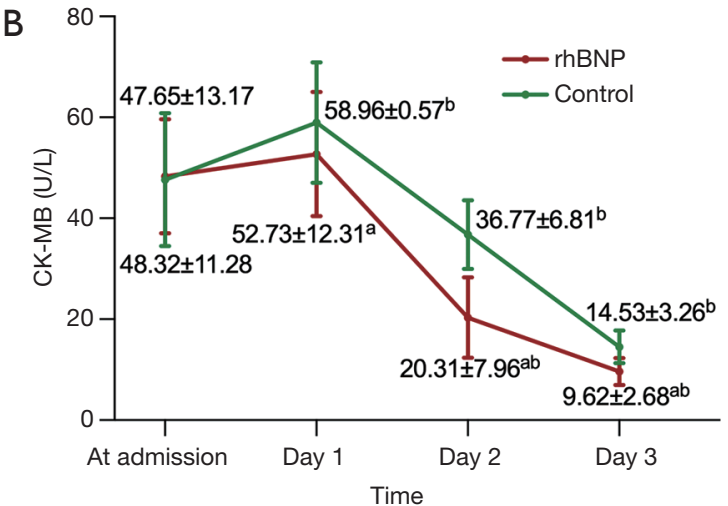

Figure 1 Comparison of cTnI (A), CK-MB (B), and ET-1 (C) between rhBNP group and control groups. ${ }^{a}, \mathrm{P}<0.05$ compared with the control group; ${ }^{\text {b }}, \mathrm{P}<0.05$ compared with the results at admission in each group. cTnI, cardiac troponin I; CK-MB, creatine kinase MB fraction; ET-1, endothelin-1; rhBNP, recombinant human brain natriuretic peptide.

at admission (Figure 2). After 3 days of treatment, QTcd significantly decreased in both groups compared with that at admission, especially in the rhBNP group $(\mathrm{P}<0.05)$. After 3 days, ALD and AT II in the rhBNP group were markedly decreased compared with those at admission $(\mathrm{P}<0.05)$. Compared with the control group, the levels of QTcd, ALD and AT II in the rhBNP group were significantly lower at day 3 after treatment $(\mathrm{P}<0.05)$.

\section{Comparisons of NT-proBNP between rbBNP and control group}

Compared with the NT-proBNP at admission, NTproBNP in the rhBNP group showed significant decrease after 1 day of treatment, while that in the control group began to show a significant decline after 4 days (Figure 3). Within 7 days of observation, the reduction in the rhBNP group was significantly greater than that in the control group at each timepoint $(\mathrm{P}<0.05)$.

\section{Comparisons of echocardiographic parameters between rbBNP and control group}

After 3 days of treatment, there was no significant difference in IVS between the rhBNP group and the control group $(\mathrm{P}=0.684$, Figure 4). However, LVEF, SV and LVEDD in the rhBNP group were all greater than those in the control group $(\mathrm{P}<0.05)$. When the follow-ups of echocardiographic parameters were extended to 1 month, all the above four parameters were similar between the rhBNP group and control group.

\section{Comparison of MACE between the rbBNP and control groups}

MACE was reported in $19(14.07 \%)$ of 135 patients in the 
A
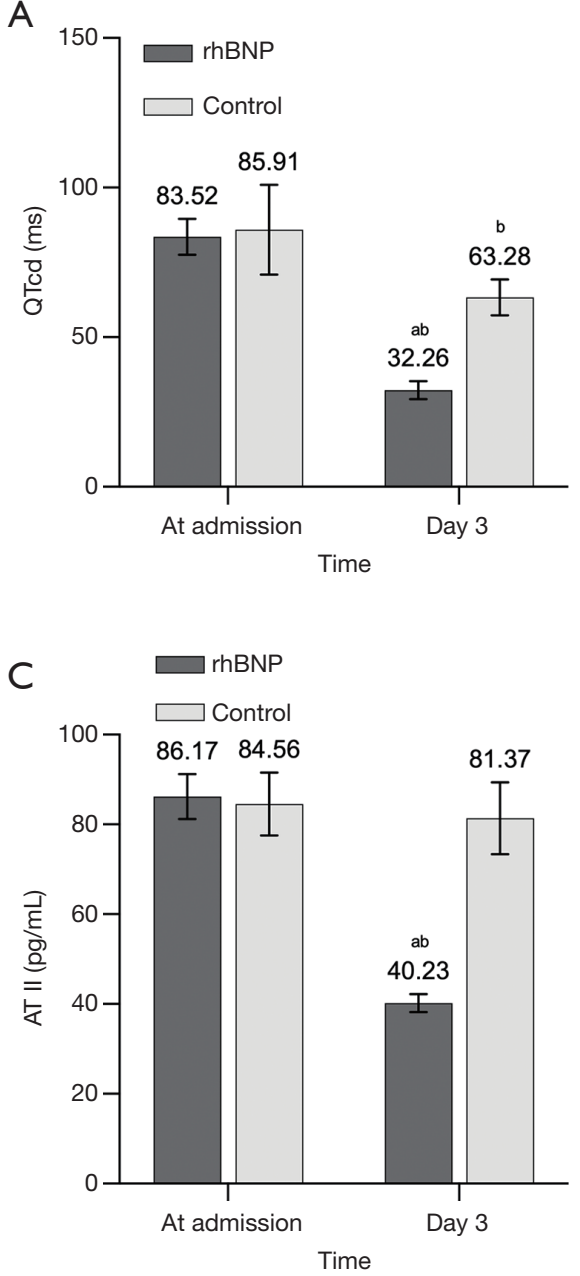

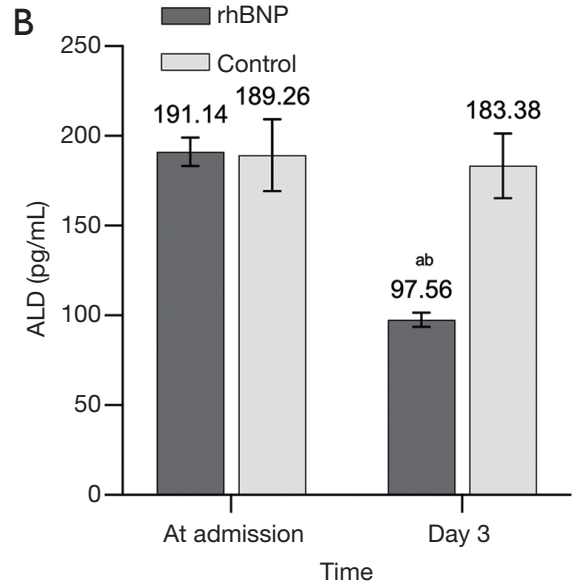

Figure 2 Comparison of QTcd (A), ALD (B), and AT II (C) between rhBNP group and control group. ${ }^{\text {a }}, \mathrm{P}<0.05$ compared with the control group; ', $\mathrm{P}<0.05$ compared with the results at admission in each group. QTcd, corrected QT dispersion; ALD, aldosterone; AT II, angiotensin II; rhBNP, recombinant human brain natriuretic peptide.

rhBNP group within 1 month after treatment, including eight acute left heart failure, four AMI, six malignant arrhythmia, and one sudden cardiac death. The incidence of MACEs in the rhBNP group was significantly lower than that in the control group $(44.12 \%, \mathrm{P}<0.05$; Table 2$)$.

\section{Discussion}

This study was conducted in patients with ACOP and high risk of cardiac events. We found that rhBNP could protect the injured myocardium of patients with ACOP, reduce the injury of $\mathrm{CO}$ on heart, and reduce the occurrence incidence of MACE.

Ding et al. developed a ischemia-reperfusion model in rabbits and found that rhBNP could protect the ventricular myocytes, reduce the area of myocardial necrosis, and significantly reduce the incidence of ischemia-reperfusion arrhythmia (14). Kamakura et al. gave rhBNP intravenous treatment to patients with AMI in the early stage of disease, and found that rhBNP could inhibit myocardial remodeling, improve hemodynamic function, reduce the activity of neuroendocrine system, and improve the survival rate of patients 1 month after treatment (15). In this study, rhBNP was given as early intervention to patients with ACOP and high risk of cardiac events. It was found that the levels of cTnI and CK-MB in the rhBNP group were lower than those in the control group at day 1, 2, and 3 after treatment. After 3 days of treatment, the LVEDD in the rhBNP 


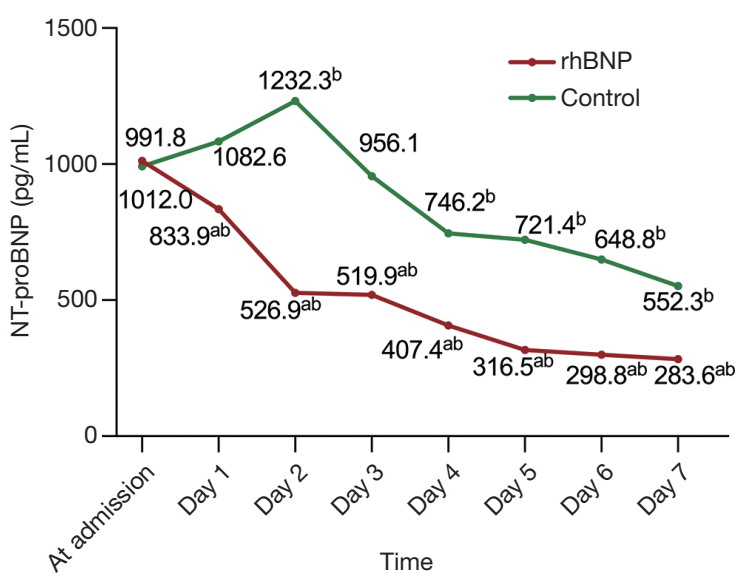

Figure 3 Comparison of NT-proBNP between rhBNP and control group. ${ }^{a}, \mathrm{P}<0.05$ compared with the control group; ${ }^{\mathrm{b}}$, $\mathrm{P}<0.05$ compared with the results at admission in each group. NTproBNP, amino-terminal pro-brain natriuretic peptide; rhBNP, recombinant human brain natriuretic peptide.

group was significantly lower than that in the control group, indicating that the treatment of rhBNP reduced the necrosis of cardiomyocytes caused by hypoxia and inhibited myocardial remodeling.

Vascular endothelium can secrete many factors and participate in immune and inflammatory reactions (16). BNP is an important hormone secreted by vascular endothelium. ET-1 is a marker of vascular endothelial function, which can reflect the grade of vascular endothelial injury and is an important vasoconstrictor (17). It can promote the opening of calcium channel, stimulate calcium ion flow into cells, and then injury cardiomyocytes. After anoxia and ischemia caused by ACOP, endothelial cells secrete a large amount of ET-1, which causes circulatory disturbance and further aggravates the cardiac afterload. ET-1, AT II and ALD play an important role in maintaining the balance of cardiac function and hemodynamics. When CO enters the body, it stimulates sympathetic nerves, activates the RAAS system, causes strong vasoconstriction and reabsorption of water and sodium, which in turn further affects the heart and forms a vicious circle (18). BNP can antagonize the RAAS and SNS system. In this study, we found that the levels of ET-1, ALD and AT II were significantly be reduced after early administration of rhBNP compared with the control group, and the vasoconstrictors produced in the body were significantly reduced after poisoning.

QTcd is the difference between the longest QT interval and the shortest QT interval on electrocardiogram (ECG), and it is an indicator to reflect whether the electrical instability of myocardium and ventricular repolarization are synchronized. When the myocardium is injured, QTcd increases. It is greatly affected by heart rate, so heart rate correction is needed to more accurately reflect the difference change of QT interval. The prolongation of QTcd induced by ACOP may be related to the aggravation of myocardial ischemia, the inhibition of potassium ion pump function and the prolongation of repolarization time course of action potential. $\mathrm{CO}$ poisoning causes increased secretion of catecholamine, and sympathetic excitation interferes with the pacing point of the heart, the automaticity of the heart muscle and the conduction system, resulting in abnormal changes such as sinus tachycardia, bradycardia, premature beat, conduction block, nodal rhythm, atrial fibrillation and ventricular fibrillation (19). In this study, the QTcd of the rhBNP group was significantly shorter than that of the control group after 72 hours of treatment. The ejection fraction and output of each stroke of the rhBNP group were significantly improved, and the NT-proBNP improvement was more significant than that of the control group. From the observation of MACE incidence within 1 month after treatment initiation, it was found that early rhBNP treatment could reduce the incidence of acute left heart failure, malignant arrhythmia, AMI and sudden cardiac death in patients with high risk of ACOP cardiac events. Only six patients in the study group developed malignant arrhythmia, while 27 patients in the control group. It is suggested that under the intervention of rhBNP, the inflammatory swelling and hypoxia of cardiomyocytes are fully improved, cardiac function is restored, cardiac repolarization is normalized, and arrhythmia is reduced.

Vasodilatation in the Management of Acute CHF (VMAC) investigators studied 489 patients with decompensated heart failure and divided them into three groups: one group was given BNP, the other two groups were given nitroglycerin and placebo, respectively (20). It was found that continuous administration of BNP could reduce pulmonary artery wedge pressure, stabilize hemodynamic state faster than the other two groups, and improve dyspnea symptoms. After the intervention, the patients can adapt to the recommended dose of treatment without obvious adverse events. Sackner-Bernstein et al. reported that rhBNP has the risk of aggravating renal function in patients (21). After that, Feng et al. conducted a clinical study on the safety of rhBNP in 408 patients with heart failure in 58 intensive care units (ICUs) in China (22). It was found that before and after the application of rhBNP, 

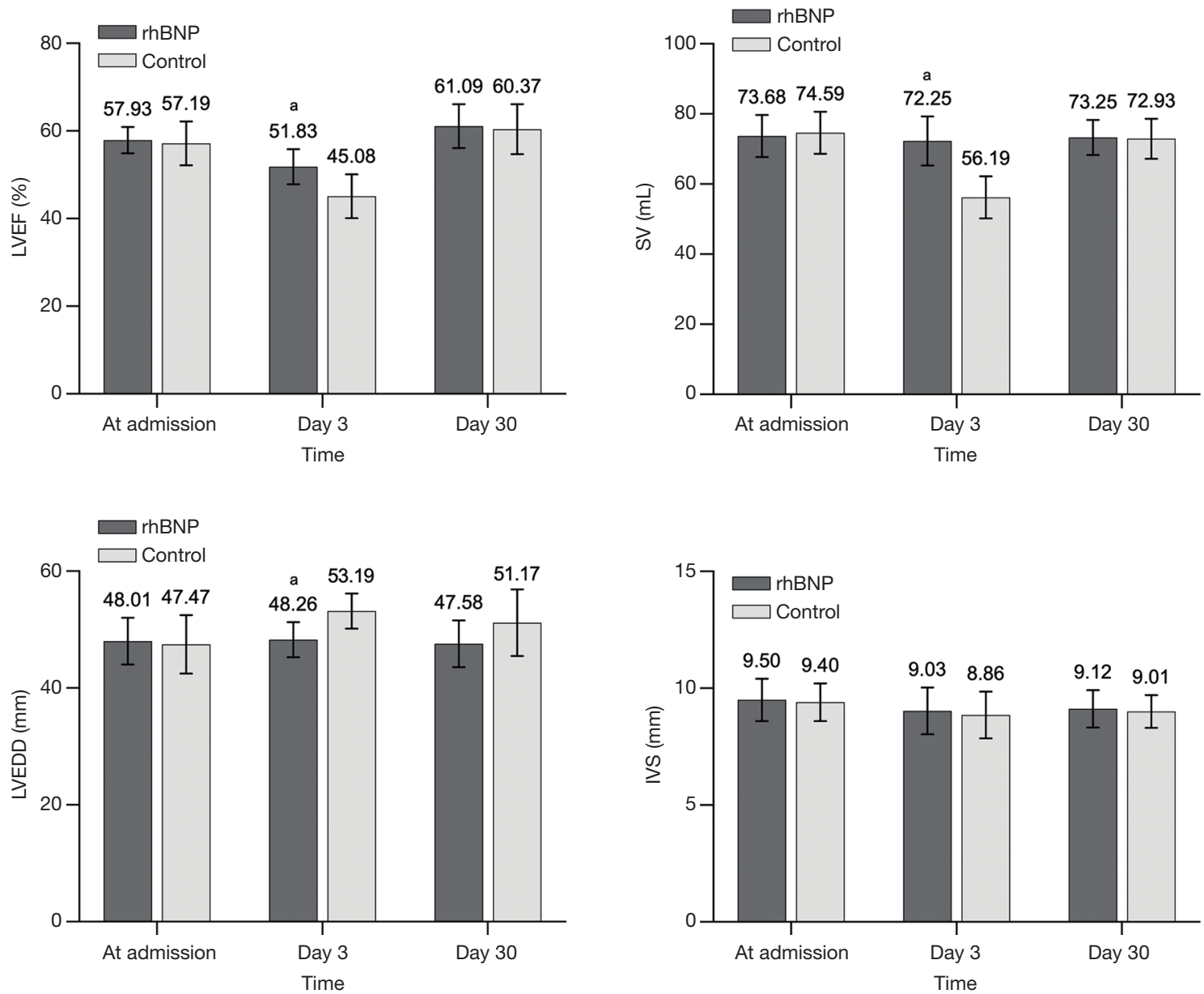

Figure 4 Comparison of echocardiographic parameters between rhBNP and control group. ${ }^{\text {a }}, \mathrm{P}<0.05$ compared with the control group. LVEF, left ventricular ejection fraction; SV, stroke output; LVEDD, left ventricular end diastolic diameter; IVS, interventricular septal thickness; rhBNP, recombinant human brain natriuretic peptide.

Table 2 Major adverse cardiac events (MACE) between the rhBNP and control groups

\begin{tabular}{|c|c|c|c|c|}
\hline MACE & rhBNP group $(n=135)$ & Control group $(n=136)$ & $\chi^{2}$ & $\mathrm{P}$ \\
\hline Acute left heart failure & $8(5.93)$ & $23(16.91)$ & 6.129 & 0.019 \\
\hline Acute myocardial infarction & $4(2.96)$ & $7(5.15)$ & 3.319 & 0.100 \\
\hline Malignant arrhythmia & $6(4.44)$ & $27(19.85)$ & 4.022 & 0.021 \\
\hline
\end{tabular}

Data are $\mathrm{n}(\%)$, unless otherwise specified. rhBNP, recombinant human brain natriuretic peptide.

no deterioration trend was found in creatinine and urea nitrogen of patients. RhBNP can rapidly increase the urine volume, play a certain protective role in the renal function of patients undergoing heart surgery, which confirmed that
rhBNP is safe and effective.

This study showed that rhBNP can protect the injured myocardium of patients with ACOP, reduce the injury of $\mathrm{CO}$ on heart, decrease the occurrence of MACE, maximize 
the benefits of patients, and provide a new basis for the treatment of patients with ACOP and high risk of cardiac events.

\section{Acknowledgments}

We thank all patients and their families.

Funding: None.

\section{Footnote}

Reporting Checklist: The authors have completed the STROBE reporting checklist. Available at http://dx.doi. org/10.21037/cdt-20-591

Data Sharing Statement: Available at http://dx.doi. org/10.21037/cdt-20-591

Conflicts of Interest: All authors have completed the ICMJE uniform disclosure form (available at http://dx.doi. org/10.21037/cdt-20-591). The authors have no conflicts of interest to declare.

Ethical Statement: The authors are accountable for all aspects of the work in ensuring that questions related to the accuracy or integrity of any part of the work are appropriately investigated and resolved. The study was conducted in accordance with the Declaration of Helsinki (as revised in 2013). The study was approved by institutional ethics committee board of Harrison International Peace Hospital (Number: 2017-0-118) and informed consent was taken from all the patients.

Open Access Statement: This is an Open Access article distributed in accordance with the Creative Commons Attribution-NonCommercial-NoDerivs 4.0 International License (CC BY-NC-ND 4.0), which permits the noncommercial replication and distribution of the article with the strict proviso that no changes or edits are made and the original work is properly cited (including links to both the formal publication through the relevant DOI and the license). See: https://creativecommons.org/licenses/by-nc-nd/4.0/.

\section{References}

1. Rose JJ, Wang L, Xu Q, et al. Carbon Monoxide Poisoning: Pathogenesis, Management, and Future Directions of Therapy. Am J Respir Crit Care Med
2017;195:596-606.

2. Kao HK, Lien TC, Kou YR, et al. Assessment of myocardial injury in the emergency department independently predicts the short-term poor outcome in patients with severe carbon monoxide poisoning receiving mechanical ventilation and hyperbaric oxygen therapy. Pulm Pharmacol Ther 2009;22:473-7.

3. Jeremias IC, Victorino VJ, Machado JL, et al. The Severity of Cecal Ligature and Puncture-Induced Sepsis Correlates with the Degree of Encephalopathy, but the Sepsis Does Not Lead to Acute Activation of Spleen Lymphocytes in Mice. Mol Neurobiol 2016;53:3389-99.

4. Cha YS, Kim H, Lee Y, Kwon W, et al. Evaluation of relationship between coronary artery status evaluated by coronary computed tomography angiography and development of cardiomyopathy in carbon monoxide poisoned patients with myocardial injury: a prospective observational study. Clin Toxicol (Phila) 2018;56:30-6.

5. Sudoh T, Kangawa K, Minamino N, et al. A new natriuretic peptide in porcine brain. Nature 1988;332:78-81.

6. Morisawa D, Hirotani S, Oboshi M, et al. Combination of hypertonic saline and low-dose furosemide is an effective treatment for refractory congestive heart failure with hyponatremia. J Cardiol Cases 2014;9:179-82.

7. O'Connor CM, Starling RC, Hernandez AF, et al. Effect of nesiritide in patients with acute decompensated heart failure. N Engl J Med 2011;365:32-43.

8. Dilić M, Nalbantic AD, Arslanagic A, et al. Biphasic and monophasic pattern of brain natriuretic peptide release in acute myocardial infarction. Coll Antropol 2011;35:155-9.

9. Stryjewski PJ, Nessler B, Cubera K, et al. Natriuretic peptides. History of discovery, chemical structure, mechanism of action and the removal routes. Basis of diagnostic and therapeutic use. Przegl Lek 2013;70:463-7.

10. Li N, Jin HX, Song Z, et al. Protective effect of recombinant human brain natriuretic peptide on acute renal injury induced by endotoxin in canines. Cell Biochem Biophys 2014;70:1317-24.

11. Dres M, Teboul JL, Monnet X. Weaning the cardiac patient from mechanical ventilation. Curr Opin Crit Care 2014;20:493-8.

12. Diagnostic Criteria of Occupational Acute Carbon Monoxide Poisoning GBZ23-200 2002. Available online: http://www.nhc.gov.cn/wjw/pyl/201212/33932.shtml

13. Prasad M, Sara J, Widmer RJ, et al. Triglyceride and Triglyceride/ HDL (High Density Lipoprotein) Ratio Predict Major Adverse Cardiovascular Outcomes in Women With Non-Obstructive Coronary Artery Disease. 
J Am Heart Assoc 2019;8:e009442.

14. Ding C, Guo J, Wang DM, et al. Influence of recombinant human brain natriuretic peptide on current of ventricular myocyte L-type calcium channels in rabbits after myocardial ischemia-reperfusion. Chinese Journal of Evidence-Based Cardiovascular Medicine 2013;5:73-5.

15. Kamakura T, Kawakami R, Nakanishi M, et al. Efficacy of out-patient cardiac rehabilitation in low prognostic risk patients after acute myocardial infarction in primary intervention era. Circ J 2011;75:315-21.

16. Krüger-Genge A, Blocki A, Franke RP, et al. Vascular Endothelial Cell Biology: An Update. Int J Mol Sci 2019;20:4411.

17. Brewster LM, Garcia VP, Levy MV, et al. Endothelin-1induced endothelial microvesicles impair endothelial cell function. J Appl Physiol (1985) 2020;128:1497-505.

18. Cachofeiro V, Miana M, de Las Heras N, et al. Aldosterone and the vascular system. J Steroid Biochem Mol Biol 2008;109:331-5.

Cite this article as: $\mathrm{Li} \mathrm{N}$, Gao X, Wang W, Wang P, Zhu B. Protective effects of recombinant human brain natriuretic peptide on the myocardial injury induced by acute carbon monoxide poisoning. Cardiovasc Diagn Ther 2020;10(6):17851794. doi: $10.21037 / \mathrm{cdt}-20-591$
19. Lyu T, Zhao Y, Zhang T, et al. Natriuretic peptides as an adjunctive treatment for acute myocardial infarction: insights from the meta-analysis of 1,389 patients from 20 trials. Int Heart J 2014;55:8-16.

20. Publication Committee for the VMAC Investigators (Vasodilatation in the Management of Acute CHF). Intravenous nesiritide vs nitroglycerin for treatment of decompensated congestive heart failure: a randomized controlled trial. JAMA 2002;287:1531-40.

21. Sackner-Bernstein JD, Skopicki HA, Aaronson KD. Risk of worsening renal function with nesiritide in patients with acutely decompensated heart failure. Circulation 2005;111:1487-91.

22. Feng X, Wu Y, Meng Y, et al. Efficacy and safety of intravenous recombinant human brain natriuretic peptide in patients with severe heart failure: a prospective multicenter clinical study. Zhonghua Wei Zhong Bing Ji Jiu Yi Xue 2017;29:520-4. 